\title{
Thermodynamic properties and phase stability of wadsleyite II
}

\author{
Kamil Tokár · Paweł T. Jochym • Przemysław Piekarz $\cdot$ \\ Jan Lażewski • Małgorzata Sternik • Krzysztof Parlinski
}

Received: 27 July 2012/ Accepted: 12 January 2013/Published online: 1 February 2013

(c) The Author(s) 2013. This article is published with open access at Springerlink.com

\begin{abstract}
The thermodynamical stability of a newly observed wadsleyite II phase in the $\mathrm{Mg}_{2} \mathrm{SiO}_{4}$ system is studied by the density functional theory. The wadsleyite II equation of state has been derived. The phase boundaries of $\mathrm{Mg}_{2} \mathrm{SiO}_{4}$ polymorphs: wadsleyite, wadsleyite II and ringwoodite are studied using the quasi-harmonic approximation at high external pressures. Clapeyron slopes determined for wadsleyite II-ringwoodite and wadsleyite-wadsleyite II boundaries are 0.0047 and $0.0058 \mathrm{GPa} / \mathrm{K}$, respectively. It is shown that the wadsleyite II phase is not thermodynamically preferred in the pure $\mathrm{Mg}_{2} \mathrm{SiO}_{4}$ system and will probably not occur between wadsleyite and ringwoodite phases.
\end{abstract}

Keywords Wadsleyite II · Transition zone $\cdot$ Phonon density of states - Thermoelastic property $\cdot$ Phase transition . Quasi-harmonic approximation

\section{Introduction}

Wadsleyite and ringwoodite, the polymorphs of $\mathrm{Mg}_{2} \mathrm{SiO}_{4}$ are considered to be the main forms of the magnesium

Electronic supplementary material The online version of this article (doi:10.1007/s00269-013-0565-9) contains supplementary material, which is available to authorized users.

K. Tokár $(\bowtie)$

Institute of Theoretical Physics, Faculty of Physics, University of Warsaw, Hoża 69, 00-681 Warsaw, Poland

e-mail: tokar@wolf.ifj.edu.pl

P. T. Jochym - P. Piekarz - J. Łażewski - M. Sternik ·

K. Parlinski

Institute of Nuclear Physics, Polish Academy of Sciences,

Radzikowskiego 152, 31-342 Cracow, Poland orthosilicates present in the Earth's upper mantle at the depths from 410 to $660 \mathrm{~km}$ (Anderson 1989). These polymorphs are denoted as the $\beta$ - and $\gamma-\mathrm{Mg}_{2} \mathrm{SiO}_{4}$ phase, respectively. The phase transitions between these polymorphs, induced by pressure and temperature changes, lead to substantial modification of crystal structure and properties (Anderson 1967). The zone of the transformation of wadsleyite into ringwoodite lays at depth of $520-525 \mathrm{~km}$ with the corresponding pressures 17-19 GPa (Dziewonski and Anderson 1981; Ringwood 1975). That transformation may be responsible for the seismic discontinuities observed in the mantle (Shearer 1990; Ryberg et al. 1997).

Wadsleyite II was discovered and described in the highpressure synthesis study (Smyth and Kawamoto 1997). The crystal structure of this new phase could be regarded as a modified wadsleyite structure and an intermediate phase between wadsleyite and ringwoodite. The occurrence of wadsleyite II in wadsleyite-ringwoodite system could explain the seismic expression in the $525 \mathrm{~km}$ discontinuity (Smyth et al. 2005). The basic characteristics of this new phase were obtained previously, including the crystal structure parameters, mechanical properties and Raman modes (Smyth et al. 2005; Kleppe et al. 2006). The elasticity tensor, mechanical properties and lattice dynamics of wadsleyite II were investigated using ab initio techniques at pressures up to $25 \mathrm{GPa}$ in our previous work (Tokár et al. 2010a), and here, we concentrate on the thermodynamics of this phase.

In this paper, we calculate the stable configuration of crystal structures of all phases under consideration: $\beta$-, $\gamma-\mathrm{Mg}_{2} \mathrm{SiO}_{4}$ and wadsleyite II at high external pressures and determine their lattice dynamics using the density functional theory (DFT). The phonon density of states (PDOS) obtained with the direct method (Parlinski et al. 1997) is then used to derive the thermodynamical functions of all 
considered phases within the quasi-harmonic approximation (QHA). The equation of state for wadsleyite II obtained in this approximation enables us to estimate its basic thermoelastic properties. The thermodynamical equilibrium lines between wadsleyite, wadsleyite II and ringwoodite determine, in turn, the boundaries in the highpressure part of the $P-T$ phase diagram. The relations of the wadsleyite II phase to wadsleyite and ringwoodite are investigated by comparing the Gibbs energy of the phases in the $P-T$ diagram. Finally, we study the stability of the wadsleyite II phase relative to the other phases.

\section{Calculation details}

Crystal structure and lattice dynamics

The structure of wadsleyite denoted as $\beta-\mathrm{Mg}_{2} \mathrm{SiO}_{4}$ has the orthorhombic unit cell with the Imma (\#74) symmetry and $\mathrm{Z}=8$ formula units, while the crystal of the ringwoodite phase $\left(\gamma-\mathrm{Mg}_{2} \mathrm{SiO}_{4}\right)$ has the cubic unit cell with the $F d \overline{3} m(\# 227)$ symmetry and also $\mathrm{Z}=8$. Wadsleyite II lattice has the Imma (\#74) symmetry, and its structure is very similar to that of wadsleyite, but the $b$ lattice constant of its unit cell is approximately 2.5 times longer and the unit cell has $Z=20$ (see Fig. 1). Structure optimization with respect to the cell size and ionic positions was performed in the $1 \times 1 \times 1$ supercell for all these structures. The VASP (Kresse and Furthmüller 1996a, b) implementation of the DFT and the projector-augmented wave
(PAW) method (Blöchl 1994) has been used together with the generalized-gradient approximation (GGA) in PerdewBurke-Ernzerhof formulation (Perdew et al. 1996) applied to the exchange-correlation interaction. The cutoff energy for electron plane wave base functions has been chosen at $500 \mathrm{eV}$, and the reciprocal space was sampled over the $2 \times 2 \times 2$ Monkhorst-Pack mesh (Monkhorst and Pack 1976). The calculations were performed for the external pressure parameter from -17.5 to $50 \mathrm{GPa}$.

The comparison of the static energy dependence of the $\mathrm{Mg}_{2} \mathrm{SiO}_{4}$ phases on cell volume is shown in Fig. 2. These dependences are plotted with a 3rd-order polynomial fit. For each crystal structure, the minimum of energy corresponds to the volume at zero external pressure. The three curves are very close to each other at a volume $\mathrm{V}_{c}=63.30 \AA^{3}$ (see Fig. 2). Wadsleyite II has energy higher than wadsleyite for $V>V_{c}$ and higher than ringwoodite for $V<V_{c}$. Ringwoodite is more stable than wadsleyite and wadsleyite II for $V<V_{c}$. These relations imply that wadsleyite II is never energetically preferred in the $\mathrm{Mg}_{2} \mathrm{SiO}_{4}$ system at $T=0$. The calculated unit cell volumes are summarized in Table 1 . The calculated volumes may be overestimated, by about $2.5 \%$, due to the properties of the DFT + GGA approach as will be discussed below.

The lattice dynamics of a crystal is determined by interatomic forces present in the structure. These forces were derived using the Hellmann-Feynman theorem from the DFT calculations. In this step, the atoms have been displaced one by one from their equilibrium positions by $0.03 \AA$.
Fig. 1 Comparison of wadsleyite (top panel) and wadsleyite II incorporating iron (bottom panel) crystal structures in (101) projection plane (b horizontal, $\mathrm{O}$ red, $\mathrm{MgO}_{6}$ octahedra orange, $\mathrm{SiO}_{4}$ tetrahedra blue, $\mathrm{Mg}$ orange, Si blue, O red, Fe brown)
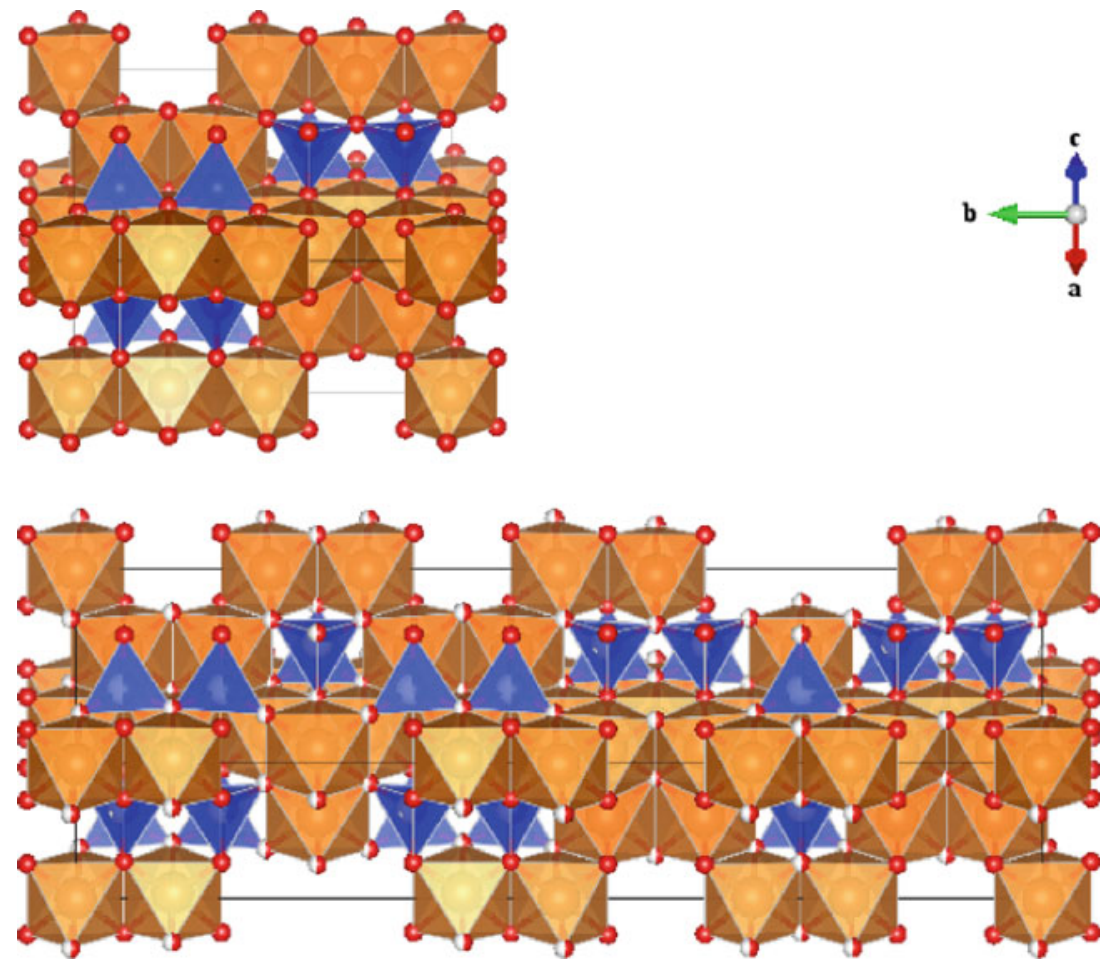


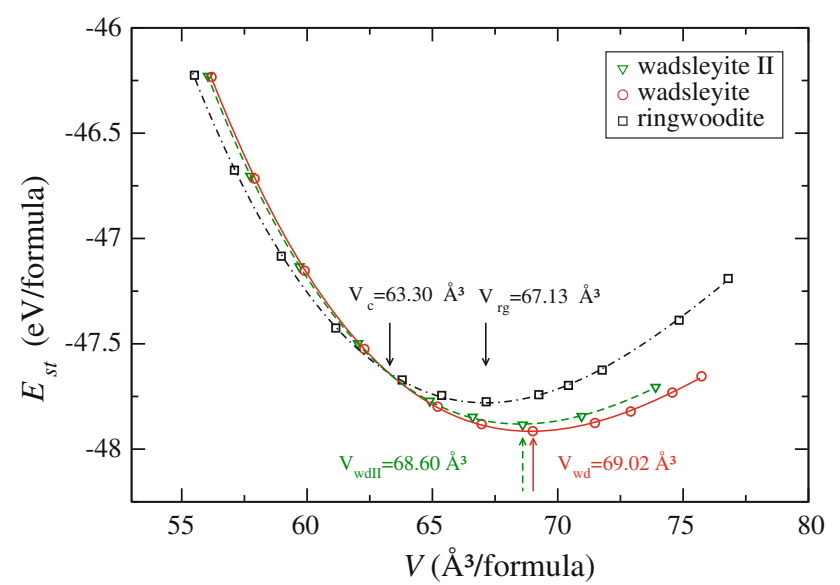

Fig. 2 The calculated static energy per formula unit of wadsleyite, wadsleyite II and ringwoodite crystal structures. The minima of energy are corresponding to the zero pressure cell volumes $V_{w d}=$ $69.02 \AA^{3}, V_{w d I I}=68.60 \AA^{3}$ and $V_{r g}=67.13 \AA^{3}$ for wadsleyite, wadsleyite II and ringwoodite, respectively. $V_{c}$ denotes a point, where all three phases have the closest static energy

Using the direct method (Parlinski et al. 1997) as implemented in the PHONON program (Parlinski 2008), a dynamical matrix has been constructed taking into account the symmetries of supercell and then diagonalized to obtain phonon dispersion relations and phonon density of states (PDOS). The distribution of vibrational states through the frequency spectrum of crystal lattice (i.e., PDOS) determines thermodynamics of the phonon system. The comparison of PDOSes calculated for $\gamma-, \beta-\mathrm{Mg}_{2} \mathrm{SiO}_{4}$, and wadsleyite II at the external pressure $P=20 \mathrm{GPa}$, which is a typical pressure scale for wadsleyite-ringwoodite phase transition, is presented in Fig. 3. Due to similarity in the crystal structure, the PDOS of wadsleyite II is very close to that of wadsleyite (shown in the same plot). From the character of phonon spectra, one can infer that all presented structures are mechanically stable at least up to this pressure.

\section{Thermodynamics}

As we noted above, the thermodynamic properties and structure stability of crystals are determined, to a large extent, by phonon spectra. PDOS generates the statistical partition sum for the phonon canonical ensemble, the thermodynamic potentials and phonon contribution to the total Helmholtz energy of the crystal. The thermodynamic potentials of the phonon system can be derived using the PDOS data obtained within the QHA approach (Kantorovich 1995; Piekarz et al. 2002; Łażewski et al. 2004; Siegel et al. 2006).

In this approach, the temperature changes of a crystal volume are replaced by the pressure dependence at zero thermodynamic temperature. A change of crystal volume under increased temperature is replaced by the change of volume due to a decrease in external pressure. A detailed method of obtaining the Helmholtz energy of phonon contribution and plotting the total Gibbs energy of equilibrated crystal structure at given pressure and temperature in QHA used here is described in (Piekarz et al. 2002; Siegel et al. 2006).

\section{Results}

\section{Equation of state}

Thermodynamical properties of wadsleyite II at pressures and temperatures of the intermediate state are important in estimation of its phase stability and phase relations in the magnesium-orthosilicate system. To our best knowledge, there were no experimental data on wadsleyite II describing thermal behavior at high pressure. The pressure changes of wadsleyite II unit cell volume at constant temperature up to $1500 \mathrm{~K}$ were estimated from the calculated Gibbs energy $G_{\mathrm{tot}}(T, P)$ of the crystal in the QHA approach using the equation of state formula:

$V(P)=\left[\frac{\partial G_{t o t}(T, P)}{\partial P}\right]_{T=\text { const. }}$.

The dependence of the cell volume on pressure at constant temperature is presented in Fig. 4 and compared with experimental data up to $10 \mathrm{GPa}$. Crystal volumes decrease monotonically with increasing external pressure, and the curve at $T=300 \mathrm{~K}$ is slightly shifted to greater volumes with respect to experimental data of

Table 1 Lattice constants and unit cell volumes obtained from the structural optimization at zero external pressure compared with the experimental data

\begin{tabular}{lllllll}
\hline Structure & Symmetry & $\mathrm{a}(\AA)$ & $\mathrm{b}(\AA)$ & $\mathrm{c}(\AA)$ & $V_{\text {cell }}\left[\AA^{3}\right]$ & References \\
\hline$\beta-\mathrm{Mg}_{2} \mathrm{SiO}_{4}(\mathrm{wd})$ & Imma & 5.747 & 11.529 & 8.331 & 552.02 & This work \\
& & 5.698 & 11.438 & 8.257 & 538.14 & Horiuchi and Sawamoto (1981) \\
$\gamma-\mathrm{Mg}_{2} \mathrm{SiO}_{4}(\mathrm{rg})$ & $F d \overline{3} m$ & 8.129 & - & - & 537.16 & This work \\
& & 8.071 & - & - & 525.75 & Hazen et al. (1993) \\
$\mathrm{Mg}_{2} \mathrm{SiO}_{4}$ (wdII) & Imma & 5.749 & 28.791 & 8.289 & 1372.00 & This work \\
& & 5.688 & 28.924 & 8.238 & 1355.31 & Smyth and Kawamoto (1997) \\
\hline
\end{tabular}


Fig. 3 Phonon density of states for wadsleyite (dashed red line), wadsleyite II (solid green line) and ringwoodite compared at the pressure $P=20 \mathrm{GPa}$
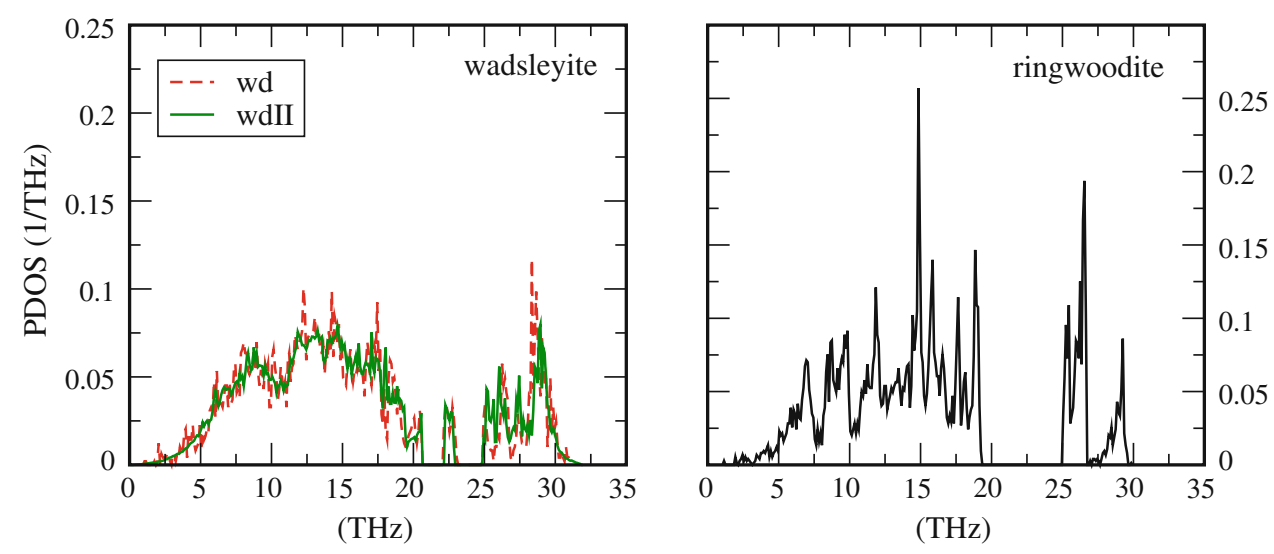

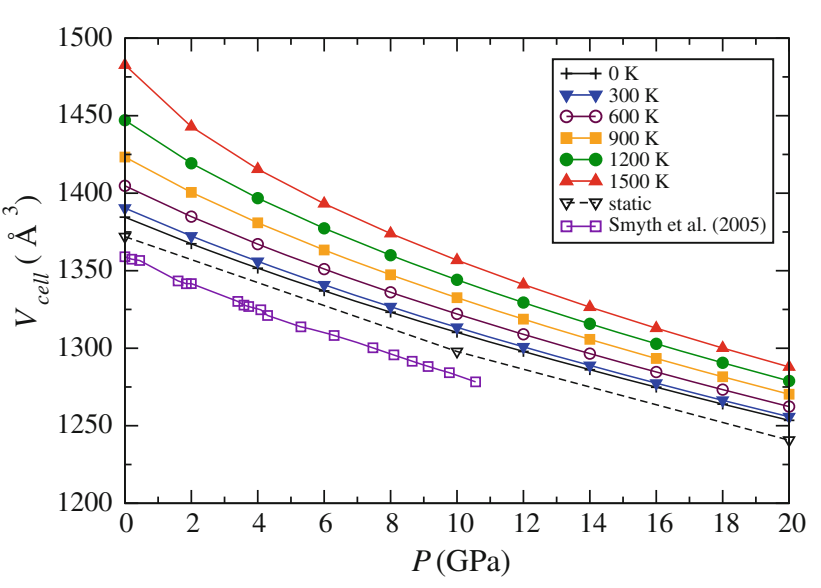

Fig. 4 Calculated cell volume isotherms of wadsleyite II at several temperatures between 0 and $1500 \mathrm{~K}$ and for external pressure up to $20 \mathrm{GPa}$. The changes of wadsleyite II unit cell volume from static calculation are marked by black dashed line. Volume dependence on the pressure is compared with the data measured at ambient temperature (Smyth et al. 2005)

Table 2 Equation of state from the QHA calculation

\begin{tabular}{llll}
\hline$T[\mathrm{~K}]$ & $V_{0}\left[\AA^{3}\right]$ & $K_{T}[\mathrm{GPa}]$ & $K_{T}^{\prime}$ \\
\hline 0 & 1385 & 159.69 & 4.7 \\
300 & 1390 & 153.30 & 4.94 \\
& $1359^{\mathrm{a}}$ & $167 \pm 10^{\mathrm{a}}$ & - \\
600 & 1405 & 140.38 & 5.48 \\
900 & 1423 & 124.26 & 6.28 \\
1200 & 1447 & 103.72 & 7.58 \\
1500 & 1483 & 73.18 & 10.59 \\
\hline
\end{tabular}

Unit cell volume and bulk moduli parameters of wadsleyite II determined from the BM EOS fit at high temperature

${ }^{\text {a }}$ Experimental value of bulk modulus at ambient conditions taken from Smyth et al. (2005)

Smyth et al. (2005). Experimental cell volume at zero pressure is $1359 \AA^{3}$ (Smyth et al. 2005), and calculated values are $1385 \AA^{3}$ at $T=0 \mathrm{~K}$ and $1390 \AA^{3}$ at $T=300 \mathrm{~K}$. The cell volume of wadsleyite II at the highest measured external pressure, $10.56 \mathrm{GPa}$, is $1278 \AA^{3}$ and that calculated at $10 \mathrm{GPa}$ and $T=300 \mathrm{~K}$ is $1313 \AA^{3}$. At ambient conditions, the difference between the theoretical and experimental cell volume is less than $3 \%$.

In order to determine the thermoelastic properties of wadsleyite II, the 3rd order Birch-Murnaghan equation of state (BM EOS) was fitted to the calculated unit cell volume isotherms (see Fig. 4). The bulk modulus $K_{T}$ and its derivative $K_{T}^{\prime}$ were obtained as parameters from the BM EOS fit (as in (Tokár et al. 2010a)) for temperatures between 0 and $1500 \mathrm{~K}$ (Table 2). The bulk modulus has decreasing tendency with the temperature, whereas its derivative is increasing. Calculated bulk moduli between 0 and $300 \mathrm{~K}$ are in good accordance with the measured $167 \pm 10 \mathrm{GPa}$ value for anhydrous limit for wadsleyite II sample (Smyth et al. 2005).

Phase diagram at high pressure

To determine the phase relations between the new wadsleyite II and two other phases wadsleyite and ringwoodite, it was necessary to determine the stability regions of all phases under consideration-a complete phase diagram of the $\mathrm{Mg}_{2} \mathrm{SiO}_{4}$ system (see the Supplementary material).

A boundary between two phases in the $P-T$ plane is located at points where both phases are in thermal and mechanical equilibrium and the Gibbs energies of both phases are equal. We have tabulated the Gibbs energies as functions of temperature at constant pressures. The crossing points of two Gibbs energy isobars determine the phase equilibrium points at appropriate pressures and temperatures. The locus of such points in the $P-T$ plane defines the equilibrium boundary of the two phases. Hence, the phase boundary between the $\beta$ - and $\gamma-\mathrm{Mg}_{2} \mathrm{SiO}_{4}$ phases is described by the implicit relation

$G_{\beta}(T, P)=G_{\gamma}(T, P)$,

which is the condition of their chemical equilibrium at the same $P$ and $T$. The Clapeyron slopes have been determined 
for all phase boundaries from linear regression fit to the calculated $P-T$ points.

Phase stability of wadsleyite II

The synthesis of wadsleyite II was observed with the presence of the wadsleyite and ringwoodite phases in the sample (Smyth and Kawamoto 1997). Therefore, the phase coexistence of wadsleyite II and these phases in the $\mathrm{Mg}_{2} \mathrm{SiO}_{4}$ system has been a major subject of our research reported here. The phase boundaries between the new wadsleyite II and ringwoodite and, separately, for wadsleyite II and wadsleyite were determined using the technique of thermodynamical comparison described above. The Gibbs energies of the phases were compared in the expected range of stability in the $P-T$ diagram at $P=$ $(0-22 \mathrm{GPa})$ and $T=(0-2000 \mathrm{~K})$. From this, comparison follows that values of Gibbs energy of wadsleyite II are always larger than those of wadsleyite or ringwoodite for each point in the $P-T$ diagram as shown in Fig. 5. It is also
Fig. 5 Differences of Gibbs energies between wadsleyite II and ringwoodite and wadsleyite and ringwoodite phases $\Delta G(T, P)=G(T, P)-$ $G_{r g}(T, P)$ at constant pressure. The Gibbs energy value of wadsleyite II (green solid line) is never the lowest one of the three in the whole temperature range
Fig. 6 Thermodynamically determined phase boundaries of: wadsleyite-wadsleyite II and wadsleyite II-ringwoodite. Solid lines describe the wadsleyite II borders. The phantom boundary of wadsleyite-ringwodite is denoted by dashed line and is depicted to compare the position of wadsleyite II stability regions. An effective Gibbs energy per one atom is defined as $g(P, T)=G(P, T) / N$, where $N$ is the number of atoms in the unit cell
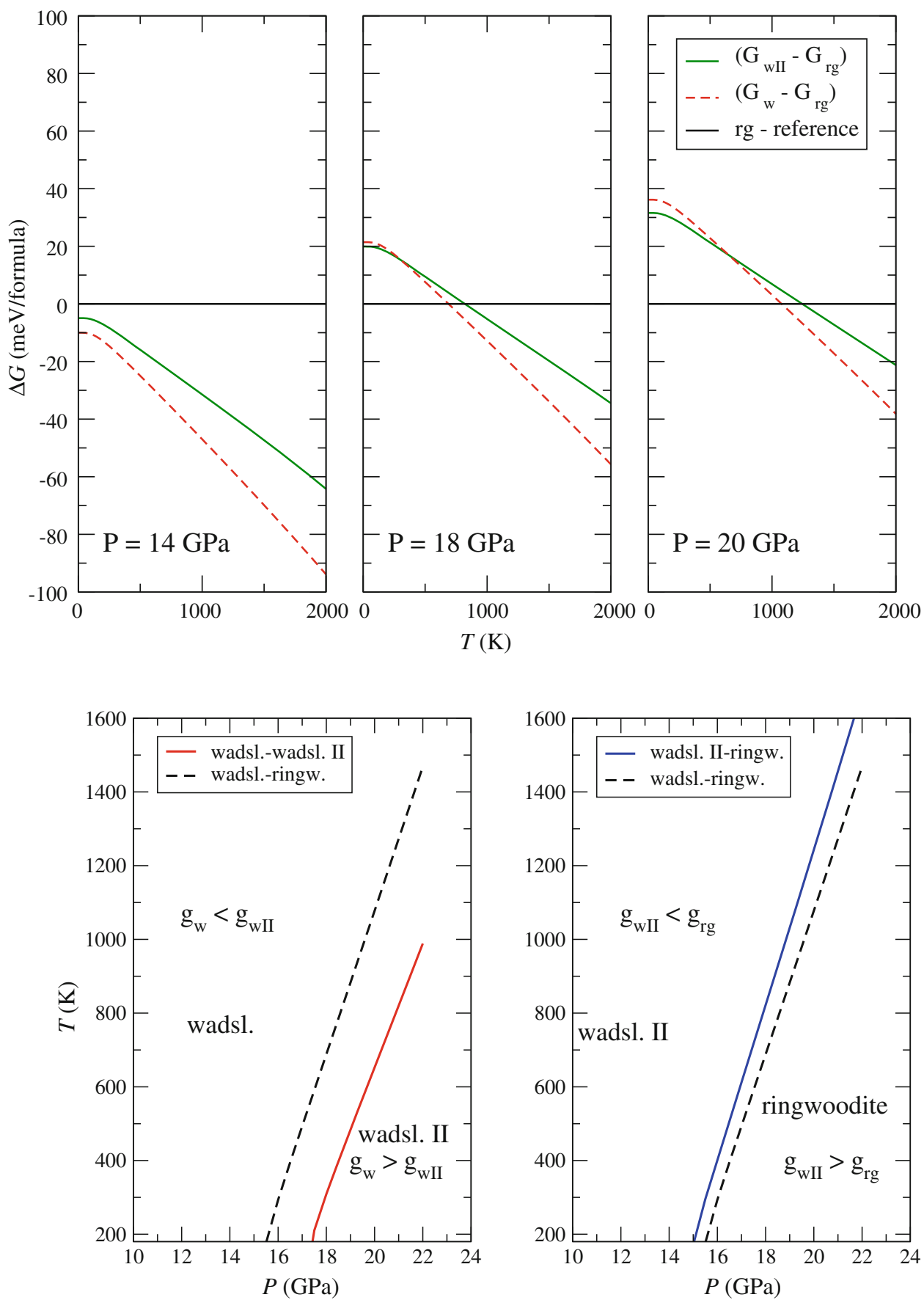
visible from positions of phase stability regions plotted in Fig. 6. The boundaries between the wadsleyite II and ringwoodite phase and between wadsleyite and wadsleyite II are depicted in the left and right panel, respectively. The regions of phase stability are positioned as in the similar case of wadsleyite-ringwoodite, which is marked with the dashed line. Comparing to ringwoodite, wadsleyite II is preferred at higher temperature and lower pressure. In the wadsleyite-wadsleyite II system, wadsleyite is preferred at higher temperature and lower pressure, whereas wadsleyite II should be more stable at higher pressure and lower temperatures. This region is positioned similarly to the ringwoodite's region of stability. These results indicate that wadsleyite II is not a preferred phase in the $\mathrm{Mg}_{2} \mathrm{SiO}_{4}$ system containing: wadsleyite, wadsleyite II and ringwoodite for any combination of pressures and temperatures. Wadsleyite II occurrence should be interpreted as an intermediate (metastable) phase in magnesium-orthosilicate system formed at specific conditions with the ringwoodite or wadsleyite. However, small content of Fe ions in the wadsleyite II may stabilize the structure as was proposed by (Smyth et al. 2005). This effect should be a subject of further research, since the presence of iron atoms in the magnesium-orthosilicate system influences significantly its structural and lattice dynamics properties (Derzsi et al. 2009, 2011; Tokár et al. 2010b).

\section{Conclusions}

The crystal structures of the wadsleyite, ringwoodite and wadsleyite II have been studied with the DFT up to the pressure $P=50 \mathrm{GPa}$. Their dynamical stability was confirmed by calculated PDOS, where no soft phonon frequencies were found. The equation of state of wadsleyite II has been determined, and the bulk moduli at ambient temperature are in good agreement with that of the experimental sample in anhydrous limit.

Complete phase diagram of magnesium orthosilicate with transformation into post-spinels has been reproduced using the direct method and the QHA. Both boundaries for the post-spinel transitions have negative slopes. Computed Clapeyron slopes are in good agreement with the experimental values, but the positions of the phase boundaries are shifted, mainly in temperature, with comparison to observed phase transitions lines.

Wadsleyite II phase boundaries have been determined separately for the wadsleyite and ringwoodite phases in the $P-T$ phase diagram. From comparison of Gibbs energies of all considered phases in the magnesium-orthosilicate system, it has been shown that wadsleyite II is not thermodynamically stable. Pure $\mathrm{Mg}_{2} \mathrm{SiO}_{4}$-wadsleyite II can be considered only as a metastable phase in this system.
Acknowledgments This research was supported by Marie Curie Research and Training Network under Contract No. MRTN-CT-2006035957 project c2c (crust to core) and Polish Ministry of Science and Education Grant No. 541/6.PR UE/2008/7.

Open Access This article is distributed under the terms of the Creative Commons Attribution License which permits any use, distribution, and reproduction in any medium, provided the original author(s) and the source are credited.

\section{References}

Anderson DL (1967) Phase changes in the upper mantle. Science 157:1165-1173

Anderson DL (1989) Theory of the Earth. Blackwell, Boston, 347, p 366

Blöchl PE (1994) Projector augmented-wave method. Phys Rev B 50:17953-17979

Derzsi M, Piekarz P, Jochym PT, Łażewski J, Sternik M, Oleś AM, Parlinski K (2009) Effects of Coulomb interaction on the electronic structure and lattice dynamics of the Mott insulator $\mathrm{Fe}_{2} \mathrm{SiO}_{4}$ spinel. Phys Rev B 79:205105

Derzsi M, Piekarz P, Tokár K, Jochym PT, Łażewski J, Sternik M, Parlinski K (2011) Comparative ab initio study of lattice dynamics and thermodynamics of $\mathrm{Fe}_{2} \mathrm{SiO}_{4^{-}}$and $\mathrm{Mg}_{2} \mathrm{SiO}_{4^{-}}$ spinels. J Phys Condens Matter 23:105401

Dziewonski AM, Anderson DL (1981) Preliminary reference Earth model. Phys Earth Planet Inter 25:297-356

Hazen RM, Downs RT, Finger LW, Ko J (1993) Crystal chemistry of ferromagnesian silicate spinels: evidence for $\mathrm{Mg}$-Si disorder. Am Mineral 78:1320-1323

Horiuchi H, Sawamoto H (1981) $\beta-\mathrm{Mg} 2 \mathrm{SiO} 4$; single-crystal X-ray diffraction study. Am Mineral 66((5-6):568-575

Kantorovich LN (1995) Thermoelastic properties of perfect crystals with nonprimitive latices. I. General theory. Phys Rev B 51(6): 3520-3534

Kleppe AK, Jephcoat AP, Smyth JR (2006) High-pressure Raman spectroscopic studies of hydrous wadsleyite II. Am Mineral 91:1102-1109

Kresse G, Furthmüller J (1996a) Efficient iterative schemes for ab initio total-energy calculations using a plane-wave basis set. Phys Rev B 54:11169-11186

Kresse G, Furthmüller J (1996b) Efficiency of ab-initio total energy calculations for metals and semiconductors using a plane-wave basis set. Comput Mater Sci 6:15-50

Łażewski J, Jochym PT, Piekarz P, Parlinski K (2004) Quasiharmonic approach to a second-order phase transition. Phys Rev B 70: 104-109

Monkhorst HJ, Pack JD (1976) Special points for Brillouin-zone integrations. Phys Rev B 13:5188-5192

Parlinski K, Li ZQ, Kawazoe Y (1997) First-principles determination of the soft mode in cubic $\mathrm{ZrO}_{2}$. Phys Rev Lett 78:4063-4066

Parlinski K (2008) PHONON software. Cracow, Poland

Perdew JP, Burke K, Ernzerhof M (1996) Generalized gradient approximation made simple. Phys Rev Lett 77:3865-3868

Piekarz P, Jochym PT, Parlinski K, Łażewski J (2002) High-pressure and thermal properties of $\gamma-\mathrm{Mg}_{2} \mathrm{SiO}_{4}$ from first-principles calculations. J Chem Phys 117:3340-3344

Ringwood AE (1975) Composition and petrology of the Earths Mantle, Chap. 14-3. McGraw-Hill, New York

Ryberg T, Wenzel F, Egorkin AV, Solodilov L (1997) Short-period observation of the $520 \mathrm{~km}$ discontinuity in northern Eurasia. J Geophys Res 102:5413-5422 
Shearer PM (1990) Seismic imaging of upper-mantle structure with new evidence for a $520 \mathrm{~km}$ discontinuity. Nature 344(6262):121-126

Siegel A, Parlinski K, Wdowik UD (2006) Ab initio calculation of structural phase transitions in AlN crystal. Phys Rev B 74: 104-116

Smyth JR, Kawamoto T (1997) Wadsleyite II: A new high pressure hydrous phase in the peridotite- $\mathrm{H}_{2} \mathrm{O}$ system. Earth Planet Sci Lett 146(1-2):9

Smyth JR, Holl CM, Langenhorst F, Laustsen HMS, Rossman GR, Kleppe A, McCammon CA, Kawamoto T, van Aken PA (2005)
Crystal chemistry of wadsleyite II and water in the Earths interior. Phys Chem Mineral 31(10):691-705

Tokár K, Jochym PT, Parlinski K, Łażewski J, Piekarz P, Sternik M (2010) DFT study of structure stability and elasticity of wadsleyite II. J Phys Condens Matter 22:145402

Tokár K, Piekarz P, Derzsi M, Jochym PT, Łażewski J, Sternik M, Oleś AM, Parlinski K (2010) Electronic and optical properties of the $\mathrm{Mg}_{2-x} \mathrm{Fe}_{x} \mathrm{SiO}_{4}$ spinel: from band insulator to Mott insulator. Phys Rev B 82(19):195116 